ARTICLE

https://doi.org/10.1038/s41467-020-14926-5

\title{
Simultaneous enhanced efficiency and thermal stability in organic solar cells from a polymer acceptor additive
}

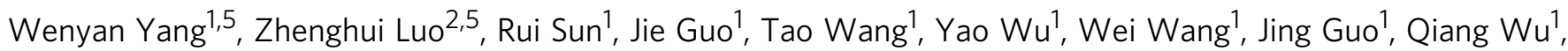
Mumin Shi', Hongneng $\mathrm{Li}^{1}$, Chuluo Yang $\mathbb{1}^{2}{ }^{2} \&$ Jie Min ${ }^{1,3,4 凶}$

The thermal stability of organic solar cells is critical for practical applications of this emerging technology. Thus, effective approaches and strategies need to be found to alleviate their inherent thermal instability. Here, we show a polymer acceptor-doping general strategy and report a thermally stable bulk heterojunction photovoltaic system, which exhibits an improved power conversion efficiency of $15.10 \%$. Supported by statistical analyses of device degradation data, and morphological characteristics and physical mechanisms study, this polymer-doping blend shows a longer lifetime, nearly keeping its efficiency $(t=800 \mathrm{~h})$ under accelerated aging tests at $150{ }^{\circ} \mathrm{C}$. Further analysis of the degradation behaviors indicates a bright future of this system in outer space applications. Notably, the use of polymer acceptor as a dual function additive in the other four photovoltaic systems was also confirmed, demonstrating the good generality of this polymer-doping strategy.

\footnotetext{
${ }^{1}$ The Institute for Advanced Studies, Wuhan University, 430072 Wuhan, China. ${ }^{2}$ Hubei Key Lab on Organic and Polymeric Optoelectronic Materials, Department of Chemistry, Wuhan University, 430072 Wuhan, China. ${ }^{3}$ Beijing National Laboratory for Molecular Sciences, 100190 Beijing, China. ${ }^{4}$ Key Laboratory of Materials Processing and Mold, Zhengzhou University, Ministry of Education, 450002 Zhengzhou, China. ${ }^{5}$ These authors contributed equally: Wenyan Yang, Zhenghui Luo. ${ }^{凶}$ email: min.jie@whu.edu.cn
} 
S olution-processed organic solar cells (OSCs) have attracted considerable attention because of their advantages, such as lightweight and flexible substrates, and colorful and easy to mass produce ${ }^{1-5}$. Particularly, non-fullerene acceptors (NFAs) have been successfully applied to high-performance OSCs and have shown promising development in the past few years ${ }^{6-8}$. The power conversion efficiencies (PCEs) of over $16 \%$ have been reported for non-fullerene OSCs based on donor polymers ${ }^{9-11}$. Despite the great achievements in improving efficiency, commercialization of large-scale OSCs is limited due to their relatively low environmental stability ${ }^{8,12-16}$. Of note is that many environmental issues (e.g., heat, oxygen, light, and humidity) have been identified as main factors of device degradation ${ }^{17}$. These parameters should be properly addressed to meet the requirements of practical outdoor application of OSCs.

It is well established that device efficiency is strongly dependent on the bulk heterojunction (BHJ) nanoscale morphology, to effectively achieve optimized charge generation, carrier transport, and carrier collection ${ }^{18-20}$. However, the optimized blend morphology, known as a thermally activated metastable state, is generally sensitive to high-temperature aging ${ }^{13,21}$, and easily moves toward a thermodynamic equilibrium state and thus forms a large phase separation, significantly reducing donor-acceptor $(\mathrm{D}-\mathrm{A})$ interfacial area and device efficiency $\mathrm{C}^{22,23}$. For instance, storing the blend film of polymer donor PTB7-Th (poly[4,8-bis (5-(2-ethylhexyl)thiophen-2-yl)benzo[1,2- $\left.b ; 4,5-b^{\prime}\right]$ dithiophene2,6-diyl-alt-(4-(2-ethylhexyl)-3-fluorothieno[3,4b] thiophene-)-2carboxylate-2-6-diyl)]) and fullerene acceptor PCBM (phenylC61-butyric acid methyl ester) in a $140^{\circ} \mathrm{C}$ oven for $2 \mathrm{~h}$ induces the formation of numerous clusters, reducing the device efficiency by $\sim 80 \%{ }^{24}$. In addition to fullerene-based photovoltaic systems ${ }^{12,24}$, many highly efficient NFA-based blends are also extremely sensitive to thermal stress ${ }^{13,25,26}$. These reported results suggest that the thermal instability of $\mathrm{BHJ}$ blends severely limit their outdoor applications, as these devices operating under standard conditions are obviously heated by prolonged illumination of sunlight ${ }^{27,28}$, and the operating temperature for solar panels can be as high as $50-70{ }^{\circ} \mathrm{C}$, even reaching $100^{\circ} \mathrm{C}$ in some areas $^{29}$. Although annealing effects have been extensively and deeply explored in OSCs ${ }^{12,14-16,24,29,30}$, their device efficiencies have been temperature dependent, and cannot withstand temperatures well over $120^{\circ} \mathrm{C}$ for extended periods of time.

Several effective approaches such as suitably reducing the crystallinity of photovoltaic polymers ${ }^{31,32}$ or fullerenes ${ }^{24,33}$, selecting suitable D/A pairs ${ }^{34,35}$, incorporating a "molecular lock"16,36, and cross-linking between D/A components in the active layers have been developed to solve thermal instability of $\mathrm{BHJ}$ blends ${ }^{22,37,38}$. Among them, blending host-active layers with additives emerges as a simple and general approach to achieve the optimal blend morphology, and also improves the electronic performance and environmental stability in $\mathrm{OSCs}^{39-41}$. The capability of packing motifs at elevated temperatures and preserving close intermolecular interactions is imperative but still lacking, especially for high-performance NFA systems ${ }^{9,14,42,43}$. We hypothesize that relevant conformational changes of interpenetrating networks between host donor and acceptor materials via introduction of semicrystalline conjugated polymers as additives can be suppressed at elevated temperatures.

Herein, we focus our endeavor to test this concept and solve the dilemma that $\mathrm{BHJ}$ photoactive layers are not resistant to high temperatures. In this work, an NFA molecule $\left(\left(2,2^{\prime}-\left(\left(2 Z, 2^{\prime} Z\right)\right.\right.\right.$ ((12,13-bis(2-ethylhexyl)-3,9-diundecyl-12,13dihydro-[1,2,5]thiadiazolo[3,4-e]thieno $\left[2^{\prime \prime}, 3^{\prime \prime}: 4^{\prime}, 5^{\prime}\right]$ thieno $\left[2^{\prime}, 3^{\prime}: 4,5\right]$ pyrrolo[3,2-g] thieno $\left[2^{\prime}, 3^{\prime}: 4,5\right]$ thieno[3,2-b]indole-2,10-diyl)bis(methanylylidene))bis(2chloro-6-oxo-5,6-dihydro-4H-cyclopenta[ $b]$ thiophene-4-ylidene) dimalononitrile)) (BTTT-2Cl, Fig. 1a) is developed. Optimized
BTTT-2Cl-based devices show a PCE of up to $13.83 \%$ by incorporating the commercially available polymer donor PM6 (as illustrated in Fig. 1a ${ }^{44}$. Adding a small amount $(1 \mathrm{wt} \%)$ of semicrystalline photovoltaic polymer acceptor $\mathrm{PZ}^{45}$ into the PM6: BTTT-2Cl host blend improved the crystalline morphology of active layer and enhanced its charge transport property, achieving a PCE of $15.10 \%$. In parallel, the excellent film quality effectively suppressed the thermally driven phase separation at elevated temperatures, and was thermally stable at $150{ }^{\circ} \mathrm{C}$ for over $800 \mathrm{~h}$ in the nitrogen-filled glovebox. Furthermore, this PZ1-doping blend also exhibits robust morphology against specific thermal-cycling stress conditions. Besides, the use of PZ1 employed as the dual function additive for improving photovoltaic efficiency and thermal stability was also confirmed in other four commonly used photovoltaic systems, suggesting that the polymer acceptor PZ1 is a universal solid additive for highly thermal-stable OSC applications.

\section{Results}

Material properties and film morphology. The chemical structures of the synthesized NFA material, BTTT-2Cl, and the polymer donor PM6 and acceptor-additive PZ1 are shown in Fig. 1a. The synthetic routes and the theoretically calculated molecular energy levels of BTTT-2Cl are provided in Supplementary Fig. 1. The synthetic details (Supplementary Figs. 1 and 2 for the nuclear magnetic resonance (NMR) spectra, and Supplementary Fig. 3 for the mass spectrum) are described in Supplementary Note 1 . Compared to the solution absorption spectra of BTTT-2Cl, an obvious bathochromic shift of $\sim 90 \mathrm{~nm}$ in its thin film (Supplementary Fig. 5) indicates high molecular ordering 46. The thermal properties of these three materials, including PM6, BTTT-2Cl, and PZ1, were investigated by differential scanning calorimetry. The corresponding results are provided in Supplementary Fig. 6. Only BTTT-2Cl shows single, sharp, and strong endothermic peak at the first heating (Supplementary Fig. 6a), which indicates its crystalline nature. As shown in Supplementary Fig. 6a, PM6 has slightly sharper endothermic peaks at low temperature and with small value of melting enthalpy. It indicates that PM6 possesses slightly higher crystallinity as compared to PZ1 (Supplementary Fig. 6c), which is identical to the previous results ${ }^{11,45}$. The ultraviolet-visible absorption spectra of the pristine films and the PM6:BTTT-2Cl blend films without and with $1 \mathrm{wt} \% \mathrm{PZ1}$, as shown in Fig. 1b, were explored. The absorption maxima of PM6:BTTT-2Cl blend shows a blue-shift compared to that of the BTTT-2Cl pristine film. Obviously, the addition of $1 \mathrm{wt} \%$ PZ1 into the host blend presents an obvious increase in the $0-0$ vibronic peak intensity with a slight red-shift, indicating the increasing molecular ordering and $\pi-\pi$ interactions in the nanoscale domains ${ }^{47}$. The highest occupied molecular orbital (HOMO) and lowest unoccupied molecular orbital (LUMO) energy levels of BTTT-2Cl, probed by cyclic voltammetry (CV) (Supplementary Fig. 7 and Supplementary Table 1) are -5.61 and $-3.98 \mathrm{eV}$, respectively. The energy levels of PM6 and PZ1 are presented in Fig. $1 \mathrm{c}^{48}$. It indicates that the PM6: BTTT-2Cl combination is comfortable for exciton dissociation in $\mathrm{BHJ}$ blend, as demonstrated by the photoluminescence (PL) measurements (Supplementary Fig. 8). Of note is that the PL intensity of PM6:BTTT-2Cl blend increases with the addition of $1 \mathrm{wt} \%$ PZ1, indicating that the interface area between PM6 and BTTT-2Cl decreases ${ }^{49}$.

This changed blend morphology was observed by atomic force microscopy (AFM) images (Fig. 1d) and two-dimensional (2D) grazing incidence wide-angle X-ray scattering (2D-GIWAXS) measurements (Fig. 1d), as well as their corresponding intensity profiles in the in-plane (IP) and out-of-plane (OOP) directions (Supplementary Fig. 9). The surface morphologies of AFM 
a

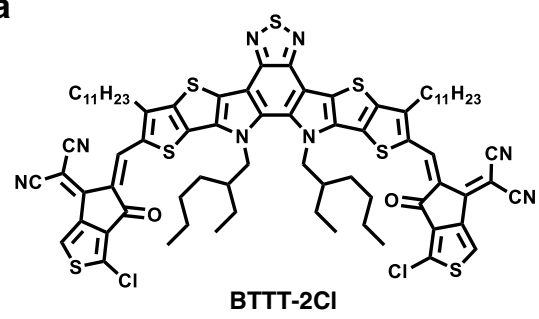

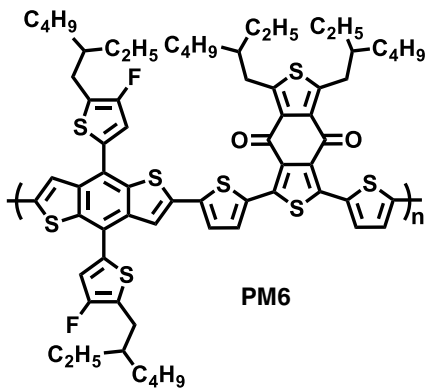

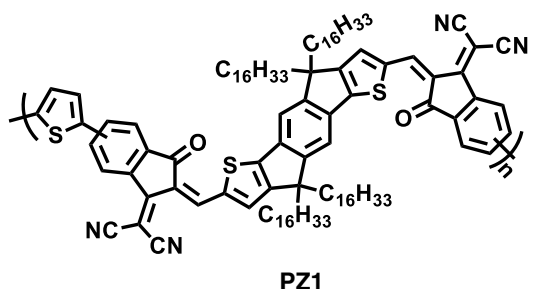

b

C

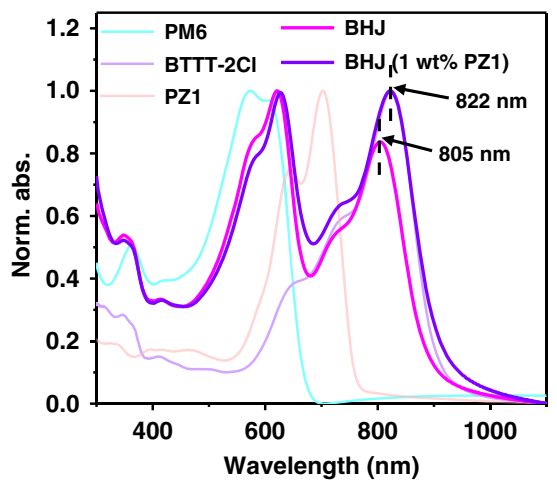

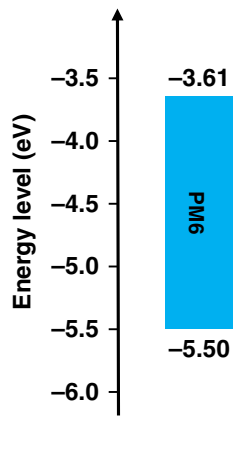

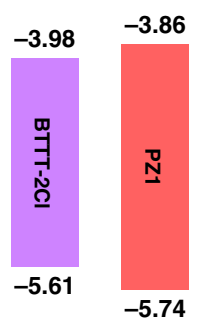

d

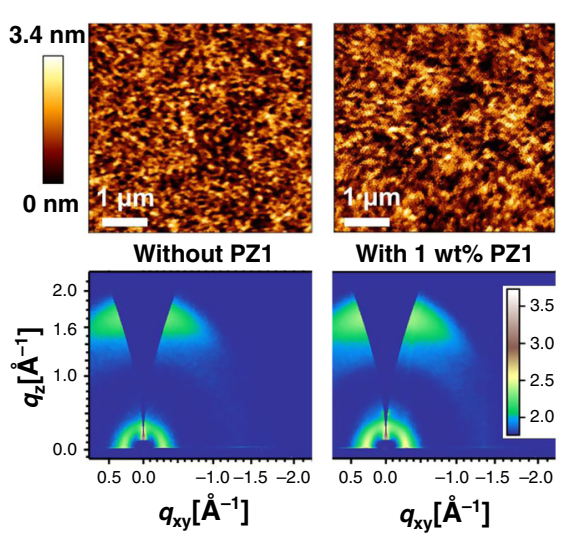

Fig. 1 Chemical structures, material characterizations, and blend morphologies. a Molecular structures of small-molecule acceptor BTTT-2Cl, polymer donor PM6, and acceptor additive PZ1. b Normalized UV-vis absorption spectra of the neat films and the PM6:BTTT-2Cl blends without and with dynamics wt\% PZ1. c Energy level diagram of PM6, BTTT-2Cl, and PZ1. d Top: Tapping-mode AFM surface scans (size: $5 \times 5 \mu m^{2}$ ) of the PM6:BTTT-2Cl blends without (a root mean square (RMS) roughness of $0.813 \mathrm{~nm}$ ) and with 1 wt\% PZ1 (an RMS roughness of $0.628 \mathrm{~nm}$ ), the $3.4-\mathrm{nm}$ scale bar applies to AFM images; bottom: 2D-GIWAXS patterns of the corresponding thin films acquired at the critical incident angle of $0.13^{\circ}$. The intensities are shown on a logarithmic scale, as indicated by the color bar.

images preliminarily demonstrated that the $1 \mathrm{wt} \%$ PZ1-doped blend shows larger interconnected domains and regions as compared to the host blend. The neat BTTT-2Cl film (Supplementary Fig. 9a) shows a strong $\pi-\pi$ stacking peak in OOP direction at $q=1.71 \AA^{-1} \quad(d \Xi \sim 3.67 \AA)$. There exists one diffraction peak in the IP direction at $q=1.47 \AA^{-1}(d \sim 4.27 \AA)$, suggesting the co-existence of two distinct structure orders ${ }^{50}$. The optimized PM6:BTTT-2Cl blend film exhibits a strong diffraction peak in the OOP direction at $q=1.70 \AA^{-1}(d \sim 3.69 \AA)$ associated with the $\pi-\pi$ stacking of BTTT-2Cl. Furthermore, the PZ1-doped host blend shows a stronger crystallization tendency confirmed by 2D-GIWAXS profiles. We calculated the crystal size by taking the full-width half-maximum (FWHM, using the Scherrer's equation) of the OOP $(010)$ peak $^{51}$. By adding $1 \mathrm{wt} \% \mathrm{PZ1}$, the crystal coherence length increased from 19.94 to $21.68 \mathrm{~nm}$, consistent with the corresponding AFM images as presented in Fig. 1d. Overall, the morphological characteristics combined with the photophysical and chemical properties of PZ1 can give a clear conclusion that a small amount of PZ1 work as a solid additive in this PM6:BTTT-2Cl system.

Photovoltaic performance of OSCs and their thermal stability. The photovoltaic devices were fabricated with a conventional architecture consisting of indium tin oxide (ITO)/poly(3,4-ethylenedioxythiophene):polystyrenesulfonate (PEDOT:PSS)/photoactive layer/perylene diimide functionalized with amino $\mathrm{N}$-oxide (PDINO)/Al. The details of device fabrication are described in the Methods section. In addition, the detailed photovoltaic performances are shown in Supplementary Figs. 10-12 for the investigated devices without and with PZ1 additives, and the detailed photovoltaic parameters are listed in Supplementary Tables 2-4. The current density-voltage $(J-V)$ curves of the corresponding best-performing devices are plotted in Fig. 2a. On the basis of a 1:1.2 blend ratio, the optimized PM6:BTTT-2Cl device exhibits a PCE of $13.80 \%\left(V_{\mathrm{oc}}\right.$ (open-circuit voltage $)=$ $0.896 \mathrm{~V}, J_{\mathrm{sc}}$ (short-circuit current density) $=23.80 \mathrm{~mA} \mathrm{~cm}^{-2}, \mathrm{FF}$ (fill factor) $=65.19 \%$ ). Here we introduced PZ1 acceptor as solid additives into the host blend for improving its device performance (Supplementary Figs. 11-13 and Supplementary Table 3). The peak efficiency, achieved at $1 \mathrm{wt} \% \mathrm{PZ1}$ yielded a high PCE of $15.10 \%\left(V_{\mathrm{oc}}=0.904 \mathrm{~V}, J_{\mathrm{sc}}=24.58 \mathrm{~mA} \mathrm{~cm}^{-2}, \mathrm{FF}=67.90 \%\right)$. Of note is that the slight voltage shift $\left(\Delta V_{\mathrm{oc}}=0.008 \mathrm{~V}\right)$ probably resulted from the high LUMO level of polymer PZ1 and the less charge carrier recombination loss, which will be mentioned below. The calculated $J_{s c}$ values obtained from the external quantum efficiency (EQE) spectra (Fig. 2b) were consistent with the measured $J_{\mathrm{sc}}$ values, as provided in Table 1 . Besides, the $J-V$ curve of the PM6:PZ1 device with a weight ratio of $1.75: 1$ is shown in Supplementary Fig. 14. The best efficiency showed a PCE of $8.27 \%\left(V_{\mathrm{oc}}=0.94 \mathrm{~V}, J_{\mathrm{sc}}=15.34 \mathrm{~mA} \mathrm{~cm}^{-2}, \mathrm{FF}=57.65 \%\right)$. It is lower than the published value $(11.2 \%)$ reported in ref. ${ }^{48}$, probably resulting from the different molecular weights of PM6 and PZ1.

We further employed space charge-limited current (SCLC) to determine the hole and electron mobilities in relevant devices (Fig. 2c and Table 1). The average hole-only mobility of PZ1doped devices $\left(\mu_{\mathrm{h}}=1.38 \times 10^{-3} \mathrm{~cm}^{2} \mathrm{~V}^{-1} \mathrm{~s}^{-1}\right.$, Supplementary Fig. 15) is higher than that of undoped devices $\left(\mu_{\mathrm{h}}=6.88 \times\right.$ $10^{-4} \mathrm{~cm}^{2} \mathrm{~V}^{-1} \mathrm{~s}^{-1}$, Supplementary Fig. 15). In addition, the 

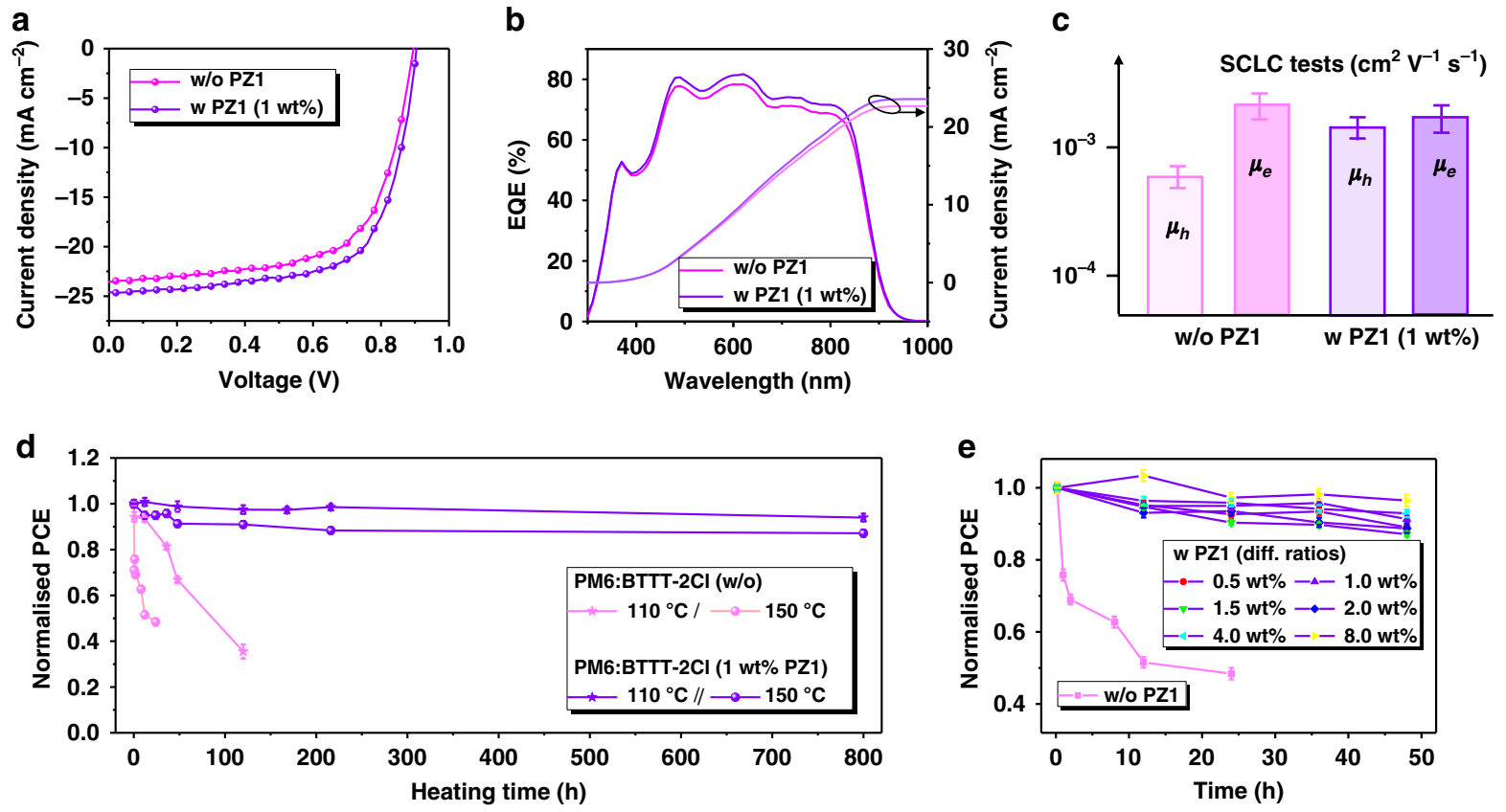

Fig. 2 Photovoltaic performance and thermal stability of the blends. a $J-V$ curves of the champion devices fabricated without and with 1 wt\% PZ1. b EQE spectra and integrated current densities from the EQE spectra. c Hole- and electron-only mobilities of relevant devices without and with 1 wt\% PZ1. d Normalized photovoltaic performance of the investigated PM6:BDTTT-2CI blends without and with 1 wt $\%$ PZ1 as a function of annealing time at $150{ }^{\circ} \mathrm{C}$ and $110{ }^{\circ} \mathrm{C}$, respectively. e Normalized PCE of corresponding blends with different PZ1 concentrations as a function of annealing time at $150{ }^{\circ} \mathrm{C}$. Source data are provided as a Source Data file.

Table 1 Detailed photovoltaic parameters and carrier mobilities of the relevant blends.

\begin{tabular}{|c|c|c|c|c|c|c|c|c|}
\hline PZ1 concentration (wt\%) & $V_{\text {oc }}(\mathrm{V})$ & $J_{s c}\left(m A c m^{-2}\right)$ & Calculated $J_{\mathrm{sc}}(\mathrm{mA} \mathrm{cm}-2)$ & FF (\%) & \multicolumn{2}{|l|}{ PCE (\%) } & \multicolumn{2}{|c|}{ Mobility $\left(\mathrm{cm}^{2} \mathrm{~V}^{-1} \mathrm{~s}^{-1}\right)$} \\
\hline 0 & 0.896 & 23.80 & 22.66 & 65.19 & $13.30 \pm 0.54$ & 13.80 & $6.88 \times 10^{-4}$ & $2.25 \times 10^{-3}$ \\
\hline
\end{tabular}

average electron-only mobility $\left(\mu_{\mathrm{e}}\right)$ values of the relevant devices without and with $1 \mathrm{wt} \% \mathrm{PZ1}$ are $2.25 \times 10^{-3}$ and $2.07 \times 10^{-3} \mathrm{~cm}^{2}$ $\mathrm{V}^{-1} \mathrm{~s}^{-1}$ (Supplementary Fig. 16), respectively. The comparable $\mu_{\mathrm{e}}$ values of the $\mathrm{PZ1}$-doped and undoped blends are probably contributed to the zinc oxide $(\mathrm{ZnO})$ morphology as well as its lower surface energy as compared to the PEDOT:PSS layer, which can affect the film formation of the active layers ${ }^{51}$. Nevertheless, combining with the more balanced transport of holes and electrons in the PZ1-doped devices, the SCLC data illustrate that charge carriers in the $1 \mathrm{wt} \%$ PZ1-doped blend can be transmitted more efficiently, which is also evidenced by the morphological characterizations (Fig. 1d). In addition, the relative recombination loss as a function of light intensity was investigated in these devices without and with $1 \mathrm{wt} \% \mathrm{PZ1}$, as plotted in Supplementary Fig. 17. It was found that adding a small amount of PZ1 into the host devices did not exhibit obvious bimolecular recombination (Supplementary Fig. 17a). In contrast, the PZ1-doped OSCs showed less trap-assisted recombination with reduced slop of $1.36 \mathrm{kT} / \mathrm{q}$ (versus $1.44 \mathrm{kT} / \mathrm{q}$ for the host devices, Supplementary Fig. 17b). The detailed analyses are provided in Supplementary Note 2. Overall, the doping of PZ1 polymer not only improves the blend morphology with suitable phase separation and large pure domains but also can reduce the carrier recombination loss and enhance the carrier transport properties in the doping blend.

Importantly, we conducted a thermal stress stability test for the PM6:BTTT-2Cl blends without and with 1 wt\% PZ1 at $110^{\circ} \mathrm{C}$ and $150^{\circ} \mathrm{C}$, respectively, in a nitrogen atmosphere. The average efficiency of the undoped blend degraded gradually from $13.30 \%$ to $6.38 \%$ and then $4.66 \%$ after constant heating at $150{ }^{\circ} \mathrm{C}$ for $24 \mathrm{~h}$ and $110^{\circ} \mathrm{C}$ for $120 \mathrm{~h}$, respectively, which represent $52 \%$ and $65 \%$ loss in efficiencies. In contrast with the host blends under the same conditions, the blends with 1 wt $\%$ PZ1 showed excellent accelerated thermal stability depicting 5\% and $12 \%$ efficiency losses after heating at $110^{\circ} \mathrm{C}$ and $150^{\circ} \mathrm{C}$ for $800 \mathrm{~h}$, as exhibited in Fig. 2d. Moreover, the devices with added PZ1 weight ratios in the range $0.5-8.0 \%$ retained $88-97 \%$ of their PCEs after $48 \mathrm{~h}$, as presented in Fig. 2e. Unsurprisingly, as shown in Supplementary Fig. 18, the above-mentioned PM6:PZ1 all-polymer photovoltaic system also retained $\sim 90 \%$ of its PCEs after $48 \mathrm{~h}$, indicating the super thermal stability at a high temperature. These results indicate that PZ1 as a solid additive has played a significant role in mitigating morphological degradation, which is mainly due to the thermally driven phase separation and BTTT-2Cl aggregations as discussed below.

Morphology and physical mechanism analysis under thermal stress. As discussed above, the addition of $\mathrm{PZ1}$ results in a favorable blend film with more obvious molecular ordering of BTTT-2Cl in comparison with the blend film without PZ1 (Fig. 1d). As shown in Fig. 3a, molecular dynamics modeling was performed to investigate the influence of the $\pi-\pi$ stacking confinement on the molecular dihedral distributions. The resulting dihedral distributions were compared to characterize the BTTT- 
a

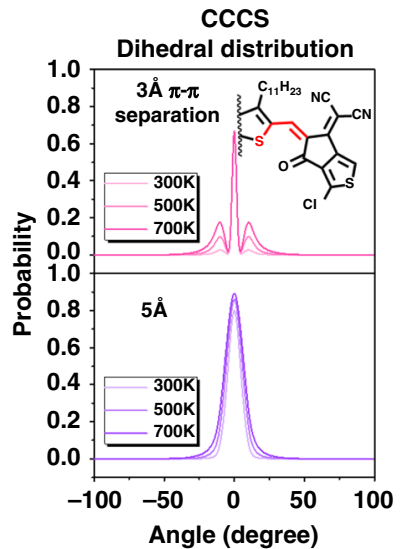

d

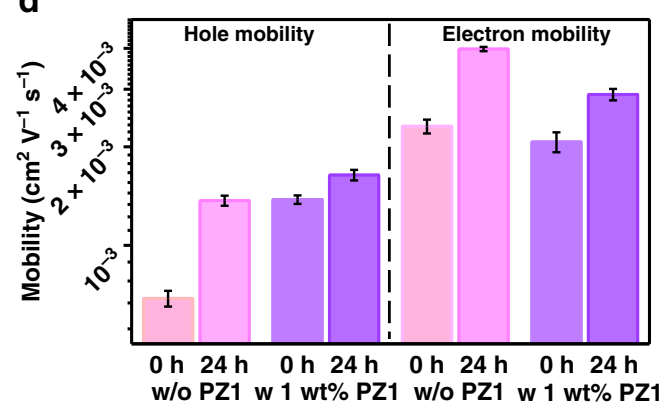

b

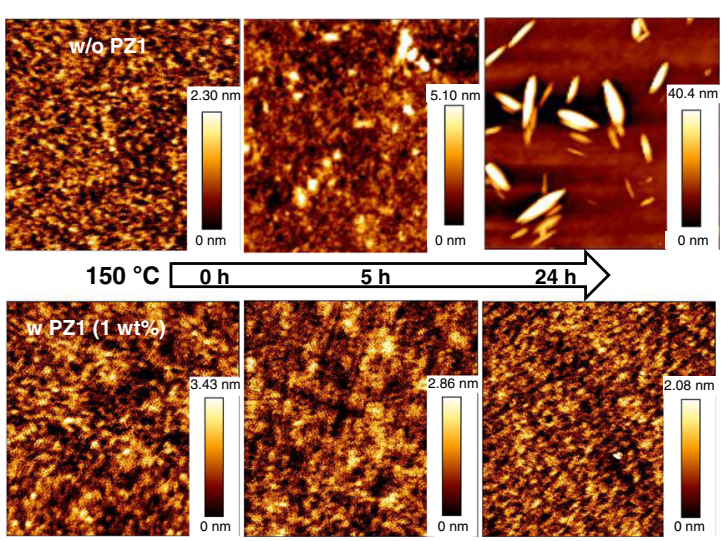

C

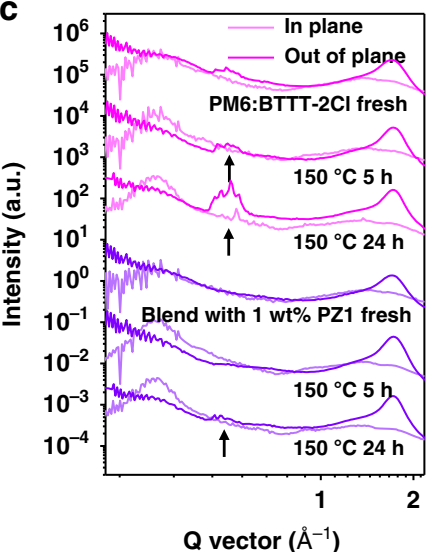

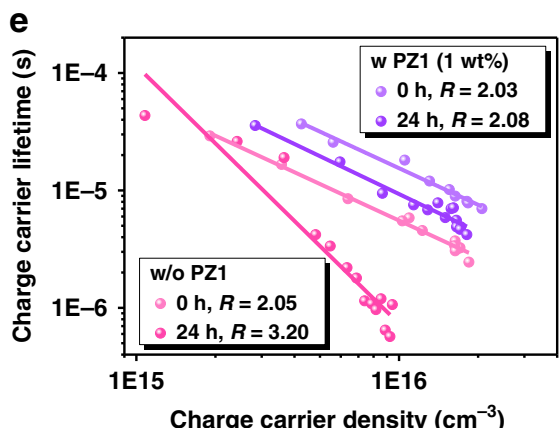

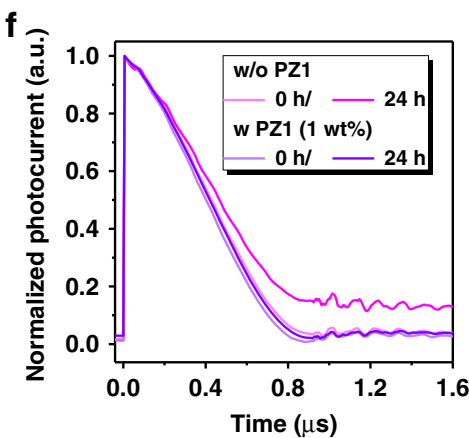

Fig. 3 Morphological characteristics and physical mechanisms of the blends. a Simulated dihedral distributions when $\pi-\pi$ confinements are confined to 3 and $5 \AA$, respectively. The distribution broadens indicate the chains twisting at different temperatures. $\mathbf{b}$ Surface topographic AFM images (size: $5 \mu \mathrm{m} \times 5 \mu \mathrm{m})$ of the undoped and PZ1-doped films under thermal stress. $\mathbf{c}$ The one-dimensional GIWAXS line curves of the corresponding blends with respect to the OOP and IP directions. $\mathbf{d}$ The hole and electron mobilities of the corresponding films as a function of heating time at $150^{\circ} \mathrm{C}$. e Charge carrier lifetime obtained from TPV measurements as a function of charge carrier density ( $n$ ) obtained from CE measurements under open-circuit conditions. $\mathbf{f}$ Normalized transient photocurrent (TPC) data for the undoped and PZ1-doped devices based on the relevant blends under thermal stress. Source data are provided as a Source Data file.

$2 \mathrm{Cl}$ reorganization dynamics. It is found that the CCCS dihedral angle, corresponding to the $\mathrm{D}-\mathrm{A}$ conformations, remained sharply peaked at $\pi-\pi$ confinements of $3.0 \AA$. At $\pi-\pi$ confinements of $5.0 \AA$, the CCCS dihedral distribution in contrast is broadened at the corresponding temperatures. These results indicate that the $\pi-\pi$ confinement exhibited by the obvious phase separation in PZ1-doped blends played a vital role in enabling temperature-insensitive molecule migration. It suggests that this PZ1-doping approach can be general to other photovoltaic systems, which will be discussed in the last section.

To understand the thermal stability of the PZ1-doped BHJ blend versus host blend, we further conducted AFM and GIWAXS measurements to investigate the morphological change of the relevant $\mathrm{BHJ}$ blends. The surface images before and after the heating at $150^{\circ} \mathrm{C}$ for 5 and $24 \mathrm{~h}$ are shown in Fig. 3b. The AFM images of the PM6:BTTT-2Cl blend as a function of annealing time at $150^{\circ} \mathrm{C}$ are shown in Supplementary Fig. 19 . The homogeneity of the PM6:BTTT-2Cl BHJ blend was altered subsequent to the heating. Many long string-like BTTT-2Cl crystals were observed in the aged blend. In contrast, the PZ1incorporated blend morphology was not significantly affected by heating. These images provide a direct evidence that PZ1 as a solid additive is very efficient in limiting the thermally induced BTTT-2Cl migration and aggregation in the blend and also reducing its phase segregation. Incorporating polymer acceptor PZ1 with long alkyl side chains into the PM6:BTTT-2Cl blend can form a strong framework and also inhibit BTTT-2Cl aggregation and crystallization. In addition, 2D-GIWAXS was also conducted to investigate the thermally induced crystallization of the BTTT-2Cl molecules in the blends (Fig. 3c, Supplementary Fig. 20, and Supplementary Table 5). The $\left(\begin{array}{lll}0 & 1\end{array}\right)$ diffraction peaks, which can be defined as parallel orientation to the substrate, exhibited prominent intensity in the PM6:BTTT$2 \mathrm{Cl}$ blend under prolonged thermal stress. In contrast, when $1 \mathrm{wt} \% \mathrm{PZ1}$ was provided into the control $\mathrm{BHJ}$ blend, the intensity growth of the GIWAXS profiles can be suppressed significantly, resulting in the low device efficiency losses at high temperatures.

Figure 3d exhibits the hole and electron mobilities of the relevant blends before and after heating at a temperature of $150^{\circ} \mathrm{C}$ for $24 \mathrm{~h}$. As presented in Supplementary Figs. 15 and 16, the hole- and electron-only mobilities of the relevant $\mathrm{BHJ}$ blends were significantly increased after thermal annealing for $24 \mathrm{~h}$, with the host blend gaining more carrier transport properties than the PZ1-doped blend. In addition to the investigation of charge transport properties in these two blends under prolonged thermal stress, we also directly determined their carrier recombination mechanisms. We combined the charge extraction (CE) and transient photovoltage (TPV) techniques to yield the charge carrier lifetime $(\tau)$ as a function of charge carrier density $(n)$ under $V_{\text {oc }}$ conditions (Supplementary Figs. 21 and 22$)^{52}$. Here, a non-geminate recombination order $R$ $(R=\lambda+1)$ was calculated via the equation $\tau=\tau_{0}\left(n_{0} / n\right)^{\lambda}$, where $\lambda$ is the so-called recombination exponent, and $\tau_{0}$ and $n_{0}$ are constants achieved from the fitting data. As exhibited in Fig. 3e, a slightly higher recombination order value $(R=2.05)$ for the undoped $\mathrm{BHJ}$ solar cell compared to the PZ1-doped device $(R=2.03)$ was observed. After $24 \mathrm{~h}$ heating, it was found that the host blend exhibited an increased recombination with an $R$ of 3.20. In 
contrast, the changes of the recombination order with an $R$ of 2.08 in the PZ1-doped device are not obvious.

The dwell time of charges in the relevant blends prior to extraction at the electrodes can be measured by transient photocurrent technique ${ }^{53}$. The reduced recombination order value demonstrated the slightly $V_{\text {oc }}$ improvement in the PZ1doped device. Figure $3 \mathrm{f}$ shows the $\mathrm{CE}$ properties of the undoped and PZ1-doped devices at $J_{\mathrm{sc}}$ condition, where the internal field equals the built-in potential $\left(V_{\mathrm{bi}}\right)$. The extraction time of the undoped solar cell was calculated to be $\tau=0.36 \mu$ s for $0 \mathrm{~h}$ and $\tau=0.47 \mu$ s for $24 \mathrm{~h}$ heating. In the case of the PZ1-doped devices before and after thermal stress, they showed very short lifetimes of $0.34 \mu \mathrm{s}$ for $0 \mathrm{~h}$ and $0.36 \mu \mathrm{s}$ for $24 \mathrm{~h}$, respectively. The more stable recombination order value and extraction lifetime suggest that the more efficient CE ability in the PZ1-doped devices as compared to that of the host devices based on the corresponding blends heating at $150{ }^{\circ} \mathrm{C}$ for $24 \mathrm{~h}$. These results are also consistent with the difference in blend morphology observed in the undoped and PZ1-doped blends (Fig. 3b, c). Briefly, the results of the molecular dynamics simulations and blend morphology investigations coupled with the analysis of carrier transport and recombination mechanisms finally underpin the photovoltaic performance deterioration of the corresponding blends under prolonged thermal stress and give detailed insight into the PZ1doping strategy responsible for optimizing and fixing the morphology of photoactive layer.

Simulation of OSCs' application in outer space. The abovediscussed thermal stability studies are focused on the long-term thermal degradation of the PZ1-doped PM6:BTTT-2Cl blend, indicating that it has the capability to successfully work after experiencing the extreme temperature at $150^{\circ} \mathrm{C}$. Since the relevant stability of photovoltaic systems under rapidly changing wide temperature range, thermal-cycling conditions is lacking, in this work we further evaluated the prolonged stability of the PZ1doped blends conducted at the simulated space environments, including Moon, Low Earth Orbital Satellite, and Mars. The testing profiles of the thermal-cycling tests were designed and defined as depicted in Fig. $4 \mathrm{a}$, c to simulate the moon, Low Earth Orbital Satellite, and Mars environments, respectively, and verify the thermal-cycling stability of the PZ1-doped PM6:BTTT-2Cl blend. Details of thermal-cycling test profiles of the blends under the different spaces are listed in Supplementary Table 6, and the experimental results are obtained as also shown in Fig. 4a, c. Of note is that the thermal stability issues are significantly investigated here. Thus, the irradiation stability of relevant active layers was not considered in the simulated environment of outer space applications. Under the simulated stress conditions based on Moon ${ }^{54}$, Low Earth Orbital Satellite, and Mars, respectively, over $90 \%$ of the original device efficiency can be retained after several thermal cycles. Obviously, the degradation behaviors of the PZ1doped blends, subjected to thermal-cycling stress conditions in which the temperature changes in a short period of time, are not obvious. Of note is that this result is relevant not only to the outer space applications but also to the real-world outdoor applications. Supplementary Figure 23 shows the durability of the stored OSCs under thermal-cycling stress between $-20^{\circ} \mathrm{C}$ and $80^{\circ} \mathrm{C}$ for $600 \mathrm{~h}$. The investigated devices exhibited great long-term stability under thermal-cycling stress even when metal electrodes and rigid glass substrates were employed in this device structure. Overall, these findings suggest that the PZ1-doped PM6:BTTT-2Cl photovoltaic system has the potential to be applied successfully in extreme environmental conditions with alternating temperatures, similar to the space applications. Based on this point, we further put forward the summary PCE estimates under different simulated conditions, which are derived as a function of the optical bandgap of photoactive layer, as provided in Fig. $4 \mathrm{~d}$. We found PCE to be $>20 \%$, which was calculated using the AM 1.5 spectrum when we simultaneously used an $\mathrm{FF}=80 \%$ and a constant $\mathrm{EQE}=85 \%$. Due to the higher solar light intensity on the surface of the Moon and Satellite (AM 0 spectrum), the device efficiency can be further improved to over $24 \%$ for $E_{\mathrm{g}} 1.28$ to $1.59 \mathrm{eV}$ (780-969 nm). Since the solar irradiance is $590 \mathrm{~W} \mathrm{~m}^{-2}$ at local noon on Mars, which is lower than that of the Earth's surface $\left(1000 \mathrm{~W} \mathrm{~m}^{-2}\right)$, the maximum evaluated PCE is $\sim 12 \%$.

Universality of the PZ1-doping strategy. Apart from the fact that PZ1 additive can efficiently enhance the thermal stability of the PM6:BDTTT-2Cl blend, here we also explored the thermal stability of PZ1 doped with four other photovoltaic systems, including PM6: $\mathrm{Y}^{46}$, J71:ITIC ${ }^{55}$, PTB7-Th:PC ${ }_{70} \mathrm{BM}^{56}$, and BDT-3T-R:PC ${ }_{70} \mathrm{BM}^{57}$ (Fig. 5a), for demonstrating the generality of our doping strategy. The detailed photovoltaic performance of the investigated devices with different contents of PZ1 additives are shown in Supplementary Figs. 24-27, and the detailed photovoltaic parameters are listed in Supplementary Tables 7-10. In addition, after heating at $150{ }^{\circ} \mathrm{C}$ for $48 \mathrm{~h}$ (Fig. 5b), the PCE of PM6:Y6 blends without PZ1 decays from $15.33 \%$ to $11.40 \%$ (Supplementary Fig. 28 and Supplementary Table 11); the PCE of J71:ITIC blends without PZ1 decays from $10.06 \%$ to $8.00 \%$ (Supplementary Fig. 29 and Supplementary Table 12); the PCE of PTB7-Th:PC ${ }_{70} B M$ blends without PZ1 decays from $8.39 \%$ to $2.65 \%$ (Supplementary Fig. 30 and Supplementary Table 13); the PCE of BDT-3T-R:PC ${ }_{70} \mathrm{BM}$ blends without PZ1 decays from $6.68 \%$ to $0.56 \%$ (Supplementary Fig. 31 and Supplementary Table 14 ), preserving $74 \%, 79 \%, 31 \%$, and $8.4 \%$ of their original average values, respectively. The addition of PZ1 improves the thermal stability of these non-fullerene and fullerene-based blends, as presented in Fig. 5b. After heating at $150{ }^{\circ} \mathrm{C}$ for $48 \mathrm{~h}$, the PCE of the $1 \mathrm{wt} \%$ PZ1-doped PM6:Y6 blends decays from 15.74 to 13.86\% (Supplementary Fig. 32 and Supplementary Table 15); the PCE of the $1 \mathrm{wt} \%$ PZ1-doped J71:ITIC blends decays from 10.76 to 9.97\% (Supplementary Fig. 33 and Supplementary Table 16); the PCE of the $1 \mathrm{wt} \%$ PZ1-doped PTB7-Th:PC ${ }_{70} \mathrm{BM}$ blends decays from 8.79 to $5.10 \%$ (Supplementary Fig. 34 and Supplementary Table 17); the PCE of the $1 \mathrm{wt} \%$ PZ1-doped BDT-3T-R:PC ${ }_{70}$ BM blends decays from 7.04 to $1.82 \%$ (Supplementary Fig. 35 and Supplementary Table 18). The PCEs of these four systems upon high temperature annealing for $48 \mathrm{~h}$ preserved $88 \%, 92 \%, 58 \%$, and $26 \%$ of their original average values, respectively. Besides, the corresponding blends with the addition of different contents of PZ1 (1.5, 2.0, and $4.0 \%$ ) also show the improved thermal stability properties (Fig. 5b). Of note is that the PCEs of these four photovoltaic systems are also improved by adding $1 \mathrm{wt} \% \mathrm{PZ} 1$ into the host blends, as exhibited in Supplementary Figs. 24-27, and summarized in Supplementary Tables 7-10. These results further demonstrated the good generality of the PZ1-doping strategy.

\section{Discussion}

In summary, we designed and synthesized an NFA, BTTT-2Cl. Using polymer PM6 as the donor, we systematically evaluated the photovoltaic properties of PM6:BTTT-2Cl solar cells with a PCE of $13.80 \%$. We further introduced a polymer acceptor $\mathrm{PZ1}$ employed as a dual functional solid additive. The addition of $1 \mathrm{wt}$ $\%$ PZ1 not only enhances $\pi-\pi$ stacking and aggregation of BTTT$2 \mathrm{Cl}$ acceptors promoting device efficiency up to $15.10 \%$ but also dramatically enhances its thermal stability at high temperatures. The morphological and physical characterizations demonstrated that PZ1 can significantly suppress the phase separation, fix the blend microstructure, and slow down the trap generation of the PZ1-doped blend. This PZ1-doped blend also exhibits great 

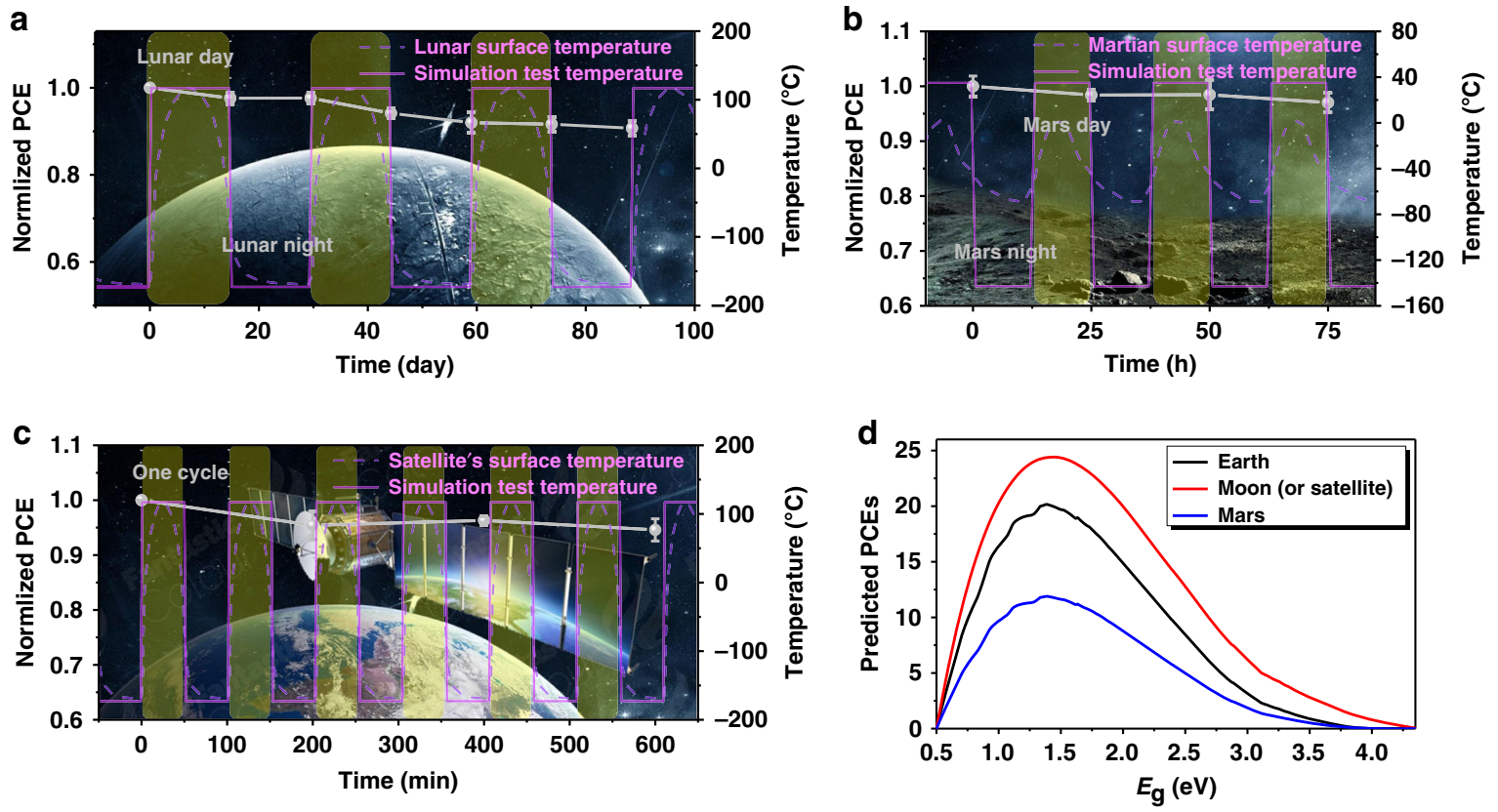

Fig. 4 Thermal cycle stability and theoretical efficiency of photovoltaic devices. Thermal cycle stability of the PZ1-doped blends (a) under the simulated thermal stress of Moon environment, $\mathbf{b}$ under the simulated thermal stress of Low Earth Orbital Satellite, and $\mathbf{c}$ under the simulated thermal stress of Mars environment. d Theoretical efficiency of non-fullerene-based solar cells with $E_{\mathrm{g}}-\mathrm{eV} \mathrm{oc}=0.5 \mathrm{eV}$ versus the lowest $E_{\mathrm{g}}$ value of the blend films, which was calculated using the $100 \%$ AM 1.5 spectrum for the Earth and 59\% AM 1.5 spectrum for the Mars 58 , and the AM 0 spectrum for the Moon and Satellite. The $E_{\mathrm{g}}$ value is determined by the broader optical absorption spectra of donor or acceptor in the film. For the purpose of calculation, an $\mathrm{FF}$ of $80 \%$ and an EQE of $85 \%$ were assumed. Source data are provided as a Source Data file.

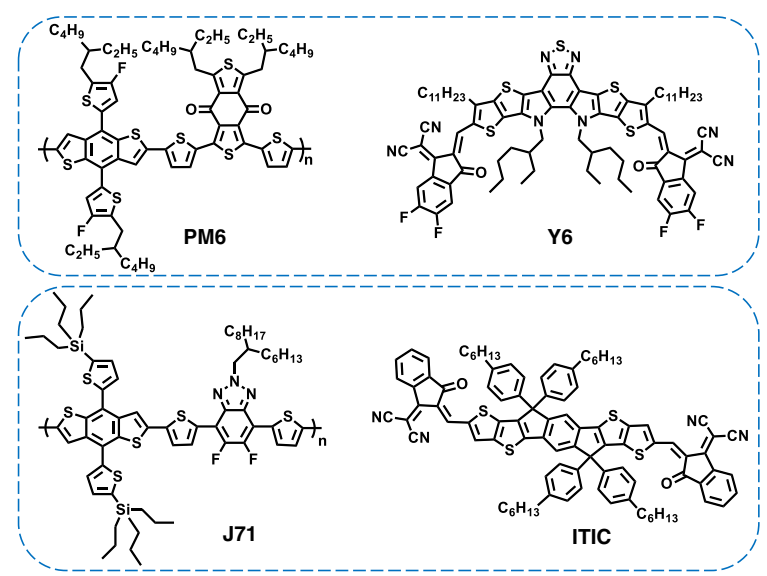

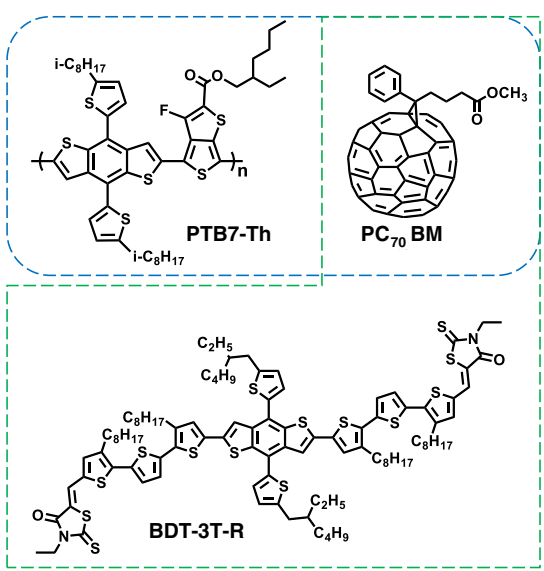

b
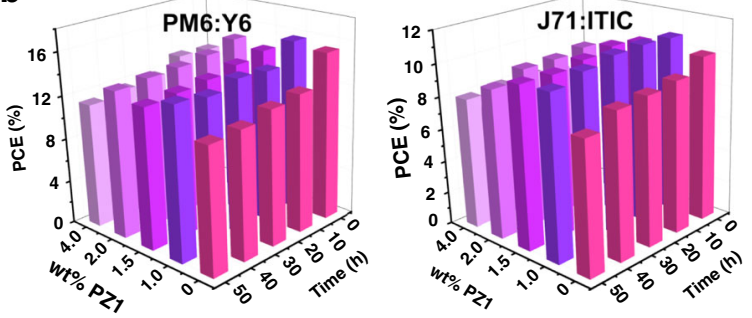

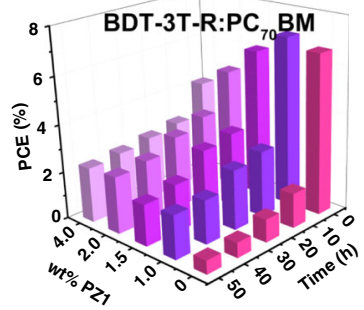

Fig. 5 Chemical structures and thermal stability of photovoltaic systems. a Molecular structures of four photovoltaic systems, including PM6:Y6, J71: ITIC, PTB7-Th:PC 70 BM, and BDT-3T-R:PC 70 BM. Normalized photovoltaic performance of investigated (b) PM6:Y6, J71:ITIC, PTB7-Th:PC 70 BM, and BDT$3 \mathrm{~T}-\mathrm{R}: \mathrm{PC} \mathrm{C}_{70} \mathrm{BM}$ blends doped by $\mathrm{PZ1}$ additives as a function of annealing time at $150^{\circ} \mathrm{C}$. 
durability under thermal-cycling stress, indicating a bright future in outer space applications. Importantly, based on the analysis of the other four photovoltaic systems, our results demonstrate that the use of polymer acceptor PZ1 as a dual function additive is a simple and general doping strategy for simultaneously improving photovoltaic performance and thermal stability of OSCs. Hence, this work provides a polymer-doping strategy to develop efficient and thermally stable organic photovoltaic systems for their perspective of industrial applications.

\section{Methods}

Materials. PTB7-Th, ITIC, and PC70BM were provided by Solarmer Materials Inc. PM6, J71, BDT-3T-R, and Y6 were synthesized according to the procedures in the literatures ${ }^{1-4}$; the synthetic route of BTTT-2Cl was shown in Supplementary Fig. 1a.

Material characterization. ${ }^{1} \mathrm{H}$ NMR spectra were measured on a Bruker DMX 400 spectrometer. The chemical shifts were reported as $\delta$ value (p.p.m.), which is relative to an internal tetramethylsilane standard. A Perkin-Elmer Lambda-35 absorption spectrometer was used to record the absorption profiles. The $\mathrm{CV}$, which was conducted on a $\mathrm{CS} 350 \mathrm{H}$ electrochemical workstation with a three-electrode system in a $0.1 \mathrm{M} \mathrm{Bu}_{4} \mathrm{NPF}_{6}$ acetonitrile solution at a scan rate of $20 \mathrm{mV} \mathrm{s}^{-1}$, was used to study the electrochemcial properties. The LUMO/HOMO energy levels $\left(E_{\mathrm{LUMO}} / E_{\mathrm{HOMO}}\right)$ can be calculated from onset reduction/oxidation potentials $\left(\phi_{\mathrm{red}} / \phi_{\mathrm{ox}}\right)$ in the CVs according to the equations of $E_{\mathrm{LUMO}} / E_{\mathrm{HOMO}}=-e$ $\left(\phi_{\text {red }} / \phi_{\mathrm{ox}}+4.8-\phi_{\mathrm{Fc} / \mathrm{Fc}}{ }^{+}\right)(\mathrm{eV})^{4}$, where $\phi_{\mathrm{Fc} / \mathrm{Fc}}{ }^{+}$is the redox potential of ferrocene/ ferrocenium $\left(\mathrm{Fc} / \mathrm{Fc}^{+}\right)$couple in the electrochemical measurement system. The relevant energy level of $\mathrm{Fc} / \mathrm{Fc}^{+}$was taken as $4.8 \mathrm{eV}$ below vacuum. In addition, DSC scans were obtained with Mettler Toledo DSC30 system with $10^{\circ} \mathrm{C} \mathrm{min}^{-1}$ heating/cooling rate in temperature range of $20-290^{\circ} \mathrm{C}$.

Fabrication and characterization of the OSCs. All the solar cells were fabricated in the conventional architecture. For the device fabrication, the ITO substrates were cleaned in toluene, water, acetone, and isopropyl alcohol. After drying, the ITO substrates were spin coated with $40 \mathrm{~nm}$ PEDOT:PSS (HC Starck, PEDOT PH4083). The photovoltaic layer was spin coated in a nitrogen glovebox from a mixed solution of PM6:BTTT-2Cl (1:1.2, wt\%) with $15 \mathrm{mg} \mathrm{mL}^{-1}$ in chloroform without and with the different concentration of PZ1 additives. In addition, the PM6:PZ1 blend was spin coated from a solution of PM6:PZ1 (1.75:1, wt\%) with $20 \mathrm{mg} \mathrm{mL}^{-1}$ in chloroform. Besides, the other four photovoltaic layers, consisting of four different photovoltaic systems as shown in Fig. 5, were dissolved in chloroform (PM6: Y6, J71:ITIC, and BDT-3T-R:PC ${ }_{70} \mathrm{BM}$ ) or ODCB (PTB7-Th:PC ${ }_{70} \mathrm{BM}$ ) with various weight ratios, and spin coated on top of the PEDOT:PSS layer. A PDINO layer via a solution concentration of $1.0 \mathrm{mg} \mathrm{mL}^{-1}$ was deposited at the top of the active layer. Finally, the top aluminum electrode of $100 \mathrm{~nm}$ thickness was evaporated in vacuum onto the cathode buffer layer at a pressure of $5 \times 10^{-6} \mathrm{mbar}$. The active area of the solar cells was $4 \mathrm{~mm}^{2}$. The device efficiencies were measured by a Keithley 2400 source meter unit under AM 1.5G $\left(100 \mathrm{~mW} \mathrm{~cm}^{-2}\right)$ irradiation from a solar simulator (Enlitech model SS-F5-3A). A monocrystalline silicon reference cell with KG5 filter was used to determine the illumination intensity of solar simulator. The EQE was measured by a Solar Cell Spectral Response Measurement System QER3011 (Enli Technology Co., Ltd.).

AFM measurements. The surface morphologies of AFM images relative to the corresponding BHJ blends were measured by a Nano Wizard 4 AFM (JPK Inc. Germany) in Qi mode.

SCLC measurements. The structure of hole-only devices was Glass/ITO/PEDOT: $\mathrm{PSS} /$ Active layer $/ \mathrm{MoO}_{3} / \mathrm{Ag}(100 \mathrm{~nm})$. In addition, the structure of electron-only mobility was Glass/ITO/ZnO/Active layer/Ca $(15 \mathrm{~nm}) / \mathrm{Ag}(80 \mathrm{~nm})$. The dark current-voltage characteristics of single carrier devices were measured and analyzed in the SCLC regime following refs. ${ }^{21,49}$

GIWAXS measurements. The 2D-GIWAXS measurements were conducted on a Xenocs-SAXS/WAXS system with X-ray wavelength of $1.5418 \AA$. The shallow incident angle scattering was collected at $0.02^{\circ}$. All samples are prepared by spincoating chloroform solutions on PEDOT:PSS-based glass substrates.

\section{Photo-induced charge carrier extraction by linearly increasing the voltage} measurements. The investigated solar cells are illuminated with a $405-\mathrm{nm}$ laser diode. A fast electrical switch was applied to isolate the device in order to prevent carrier extraction or sweep out. After the variable delay time, the switch connected the device to a function generator. In addition, current transients are recorded across the internal $50 \Omega$ resistor of our oscilloscope. To investigate the carrier mobility in the corresponding devices, the photo-CELIV (charge carrier extraction by linearly increasing the voltage) curves were conducted using different experimental conditions, differing in delay time and applied voltage.
TPV and CE measurements. For TPV measurements, a small optical perturbation was applied using a 405-nm laser diode, which was adjusted in light intensity to produce a voltage perturbation of $\Delta V_{\mathrm{o}}<10 \mathrm{mV} \ll V_{\mathrm{oc}}$. The corresponding devices were illuminated with a white-light LED (light-emitting diode) at different light intensities. The amount of charges generated by the pulse was obtained by integrating a photocurrent measurement. For CE measurements, the corresponding devices were held at a specified voltage in the dark or under different light intensities. The initial device voltage was set by a Keithley 2440 sourcemeasurement unit. In addition, a fast analog switch from Texas Instruments (TS5A23159) is used to perform the switching from the specified voltage to shortcircuit conditions. It provides a low on-state resistance $(1 \Omega)$, a quick switching time $(50 \mathrm{~ns})$, a very low charge injection $\left(<<10^{15} \mathrm{~cm}^{2} \mathrm{~V}^{-1} \mathrm{~s}^{-1}\right)$, and high off-state resistance $(>1 \mathrm{M} \Omega)$

PL measurements. The PL data and emission of relevant films were collected using a Zolix Flex One spectrometer. The PL excitation wavelength was set to $639 \mathrm{~nm}$.

Reporting summary. Further information on research design is available in the Nature Research Reporting Summary linked to this article.

\section{Data availability}

The relevant data are available from the authors upon reasonable request. In addition, the source data underlying Figs. 2c, 3d, 4a-c and Supplementary Figs. 12, 19, as well as Tables 1, 2, 3, 4, 7, 8, 9, and 10 are provided as a Source Data file.

Received: 30 October 2019; Accepted: 10 February 2020; Published online: 05 March 2020

\section{References}

1. Brus, V. V. et al. Solution-processed semitransparent organic photovoltaics: from molecular design to device performance. Adv. Mater. 31, 1900904 (2019).

2. Zhang, J., Tan, H. S., Guo, X., Facchetti, A. \& Yan, H. Material insights and challenges for non-fullerene organic solar cells based on small molecular acceptors. Nat. Energy 3, 720-731 (2018).

3. Wang, G., Adil, M. A., Zhang, J. \& Wei, Z. Large-area organic solar cells: material requirements, modular designs, and printing methods. Adv. Mater. https://doi.org/10.1002/adma.201805089 (2018).

4. Zhao, F., Wang, C. \& Zhan, X. Morphology control in organic solar cells. Adv Energy Mater. 8, 1703147 (2018).

5. Li, Y., Xu, G., Cui, C. \& Li, Y. Flexible and semitransparent organic solar cells. Adv. Energy Mater. 8, 1701791 (2018).

6. Hou, J., Inganas, O., Friend, R. H. \& Gao, F. Organic solar cells based on nonfullerene acceptors. Nat. Mater. 17, 119-128 (2018).

7. Zhang, G. et al. Nonfullerene acceptor molecules for bulk heterojunction organic solar cells. Chem. Rev. 118, 3447-3507 (2018).

8. Li, N., McCulloch, I. \& Brabec, C. J. Analyzing the efficiency, stability and cost potential for fullerene-free organic photovoltaics in one figure of merit. Energy Environ. Sci. 11, 1355-1361 (2018).

9. Xu, X. et al. Single-junction polymer solar cells with $16.35 \%$ efficiency enabled by a platinum(II) complexation strategy. Adv. Mater. 31, 1901872 (2019).

10. Fan, B. et al. Achieving over $16 \%$ efficiency for single-junction organic solar cells. Sci. China Chem. 62, 746-752 (2019).

11. Cui, Y. et al. Over $16 \%$ efficiency organic photovoltaic cells enabled by a chlorinated acceptor with increased open-circuit voltages. Nat. Commun. 10, 2515 (2019).

12. Zhang, C. et al. A top-down strategy identifying molecular phase stabilizers to overcome microstructure instabilities in organic solar cells. Energy Environ. Sci. 12, 1078-1087 (2019).

13. Speller, E. M. et al. From fullerene acceptors to non-fullerene acceptors: prospects and challenges in the stability of organic solar cells. J. Mater. Chem. A 7, 23361-23377 (2019).

14. Zhang, Y. et al. Thermally stable all-polymer solar cells with high tolerance on blend ratios. Adv. Energy Mater. 8, 1800029 (2018).

15. He, Y. et al. Evidencing excellent thermal- and photostability for singlecomponent organic solar cells with inherently built-in microstructure. $A d v$. Energy Mater. 9, 1900409 (2019).

16. Hung, K.-E. et al. Bispentafluorophenyl-containing additive: enhancing efficiency and morphological stability of polymer solar cells via handgrabbing-like supramolecular pentafluorophenyl-fullerene interactions. ACS Appl. Mater. Interfaces 9, 43861-43870 (2017).

17. Bristow, N. \& Kettle, J. Outdoor organic photovoltaic module characteristics: Benchmarking against other PV technologies for performance, calculation of 
Ross coefficient and outdoor stability monitoring. Sol. Energy Mater. Sol. Cells 175, 52-59 (2018).

18. Gurney, R. S., Lidzey, D. G. \& Wang, T. A review of non-fullerene polymer solar cells: from device physics to morphology control. Rep. Prog. Phys. 82, 036601 (2019).

19. Ye, L. et al. Miscibility-function relations in organic solar cells: significance of optimal miscibility in relation to percolation. Adv. Energy Mater. 8, 1703085 (2018).

20. Lee, H., Park, C., Sin, D. H., Park, J. H. \& Cho, K. Recent advances in morphology optimization for organic photovoltaics. Adv. Mater. 30, 1800453 (2018).

21. Min, J. et al. Side-chain engineering for enhancing the properties of small molecule solar cells: a trade-off beyond efficiency. Adv. Energy Mater. 6, 1600515 (2016).

22. Griffini, G. et al. Long-term thermal stability of high-efficiency polymer solar cells based on photocrosslinkable donor-acceptor conjugated polymers. $A d v$. Mater. 23, 1660-1664 (2011)

23. Gasperini, A., Jeanbourquin, X. A., Rahmanudin, A., Yu, X. \& Sivula, K. Enhancing the thermal stability of solution-processed small-molecule semiconductor thin films using a flexible linker approach. Adv. Mater. 27, 5541-5546 (2015)

24. Zhang, C. et al. Overcoming the thermal instability of efficient polymer solar cells by employing novel fullerene-based acceptors. Adv. Energy Mater. 7, 1601204 (2017).

25. Liao, M.-H. et al. Morphological stabilization by supramolecular perfluorophenyl-c60interactions leading to efficient and thermally stable organic photovoltaics. Adv. Funct. Mater. 24, 1418-1429 (2014).

26. Yu, R. et al. Design and application of volatilizable solid additives in nonfullerene organic solar cells. Nat. Commun. 9, 4645 (2018).

27. Xu, X. et al. Thermally stable, highly efficient, ultraflexible organic photovoltaics. Proc. Natl Acad. Sci. USA 115, 4589-4594 (2018).

28. Yang, X. et al. Suppressing thermally induced fullerene aggregation in organic solar cells by employing plastic network. J. Phys. Chem. C 122, 9843-9851 (2018).

29. Hsieh, Y. J. et al. Insights into the morphological instability of bulk heterojunction PTB7-Th/PCBM solar cells upon high-temperature aging. ACS Appl. Mater. Interfaces 9, 14808-14816 (2017).

30. Heumueller, T. et al. Morphological and electrical control of fullerene dimerization determines organic photovoltaic stability. Energy Environ. Sci. 9, 247-256 (2016).

31. Kevin Sivula, C. K. L., Barry, C. Thompson. \& Jean, M. J. F. Enhancing the thermal stability of polythiophene:fullerene solar cells by decreasing effective polymer regioregularity. J. Am. Chem. Soc. 128, 13988-13989 (2006).

32. Bertho, S. et al. Improved thermal stability of bulk heterojunctions based on side-chain functionalized poly(3-alkylthiophene) copolymers and PCBM. Sol. Energy Mater. Sol. Cells 110, 69-76 (2013).

33. Zhang, Y. et al. A simple and effective way of achieving highly efficient and thermally stable bulk-heterojunction polymer solar cells using amorphous fullerene derivatives as electron acceptor. Chem. Mater. 21, 2598-2600 (2009).

34. Kim, T., Choi, J., Kim, H. J., Lee, W. \& Kim, B. J. Comparative study of thermal stability, morphology, and performance of all-polymer fullerene-polymer, and ternary blend solar cells based on the same polymer donor. Macromolecules 50, 6861-6871 (2017).

35. Wang, W. et al. A new small molecule donor for efficient and stable all small molecule organic solar cells. Org. Electron. 70, 78-85 (2019).

36. Cheng, P. et al. Molecular lock: a versatile key to enhance efficiency and stability of organic solar cells. Adv. Mater. 28, 5822-5829 (2016).

37. Chen, C.-P., Huang, C.-Y. \& Chuang, S.-C. Highly thermal stable and efficient organic photovoltaic cells with crosslinked networks appending open-cage fullerenes as additives. Adv. Funct. Mater. 25, 207-213 (2015).

38. Feng, W. et al. Photoactive layer crosslinking in all-polymer solar cells: Stabilized morphology and enhanced thermal stability. Sol. Energy Mater. Sol. Cells 200, 109982 (2019).

39. Gasparini, N. et al. Favorable mixing thermodynamics in ternary polymer blends for realizing high efficiency plastic solar cells. Adv. Energy Mater. 9, 1803394 (2019).

40. de Zerio, A. D. \& Müller, C. Glass forming acceptor alloys for highly efficient and thermally stable ternary organic solar cells. Adv. Energy Mater. 8, 1702741 (2018).

41. Cheng, P. et al. Alloy acceptor: superior alternative to pcbm toward efficient and stable organic solar cells. Adv. Mater. 28, 8021-8028 (2016).

42. Yu, R. et al. Enhanced pi-pi interactions of nonfullerene acceptors by volatilizable solid additives in efficient polymer solar cells. Adv. Mater. 31, 1900477 (2019).

43. Cui, Y. et al. Achieving over $15 \%$ efficiency in organic photovoltaic cells via copolymer design. Adv. Mater. 31, 1808356 (2019).
44. Zhang, M., Guo, X., Ma, W., Ade, H. \& Hou, J. A large-bandgap conjugated polymer for versatile photovoltaic applications with high performance. $A d v$. Mater. 27, 4655-4660 (2015).

45. Zhang, Z. G. et al. Constructing a strongly absorbing low-bandgap polymer acceptor for high-performance all-polymer solar cells. Angew. Chem. Int. Ed. Engl. 56, 13503-13507 (2017).

46. Yuan, J. et al. Single-junction organic solar cell with over 15\% efficiency using fused-ring acceptor with electron-deficient core. Joule 3, 1140-1151 (2019).

47. Panzer, F., Bässler, H. \& Köhler, A. Temperature induced order-disorder transition in solutions of conjugated polymers probed by optical spectroscopy. J. Phys. Chem. Lett. 8, 114-125 (2016)

48. Meng, Y. et al. 11.2\% Efficiency all-polymer solar cells with high open-circuit voltage. Sci. China Chem. 62, 845-850 (2019).

49. Min, J. et al. A solution-processable star-shaped molecule for highperformance organic solar cells via alkyl chain engineering and solvent additive. Org. Electron. 14, 219-229 (2013).

50. Mai, J. et al. Hidden structure ordering along backbone of fused-ring electron acceptors enhanced by ternary bulk heterojunction. Adv. Mater. 30, 1802888 (2018).

51. Sun, R. et al. Spontaneous open-circuit voltage gain of fully fabricated organic solar cells caused by elimination of interfacial energy disorder. Energy Environ. Sci. 12, 2518-2528 (2019).

52. Sun, R. et al. A universal layer-by-layer solution-processing approach for efficient non-fullerene organic solar cells. Energy Environ. Sci. 12, 384-395 (2019).

53. Sun, R. et al. A multi-objective optimization-based layer-by-layer bladecoating approach for organic solar cells: rational control of vertical stratification for high performance. Energy Environ. Sci. 12, 3118-3132 (2019).

54. Delgado-Bonal, A. \& Martín-Torres, F. J. Solar cell temperature on Mars. Sol. Energy 118, 74-79 (2015).

55. Bin, H. et al. $11.4 \%$ Efficiency non-fullerene polymer solar cells with trialkylsilyl substituted 2D-conjugated polymer as donor. Nat. Commun. 7, 13651 (2016)

56. Yang, W. et al. Finely tuned cores in star-shaped zwitterionic molecules for interface engineering of high-performance polymer solar cells. Sol. RRL 3, 1900166 (2019).

57. Badgujar, S. et al. Highly efficient and thermally stable fullerene-free organic solar cells based on a small molecule donor and acceptor. J. Mater. Chem. A 4 , 16335-16340 (2016).

58. Malla, R. B. \& Brown, K. M. Determination of temperature variation on lunar surface and subsurface for habitat analysis and design. Acta Astronautica 107, 196-207 (2015).

\section{Acknowledgements}

This work was financially supported by the National Natural Science Foundation of China (NSFC) (Grant Nos. 21702154 and 51773157). We also thank the support of the opening project of Key Laboratory of Materials Processing and Mold and Beijing National Laboratory for Molecular Sciences (BNLMS201905).

\section{Author contributions}

W.Y.Y. and J.M. conceived and developed the ideas. W.Y.Y. designed the experiments and performed device fabrication. Z.H.L. and C.L.Y. synthesized the BTTT-2Cl. W.Y.Y. and R.S. performed TMU measurements. Jie G. carried out molecular dynamics simulation. T.W. and H.N.L. synthesized the PM6 and J71 materials. Y.W. synthesized the BDT-3T-R material. W.W. carried out CV test and analysis. Jing G. and M.M.S. performed PL measurement. Q.W. carried out the SCLC test. R.S. conducts AFM measurement. W.Y.Y. and J.M. wrote the manuscript.

\section{Competing interests}

The authors declare no competing interests.

\section{Additional information}

Supplementary information is available for this paper at https://doi.org/10.1038/s41467 020-14926-5.

Correspondence and requests for materials should be addressed to J.M.

Peer review information Nature Communications thanks the anonymous reviewer(s) for their contribution to the peer review of this work. Peer reviewer reports are available.

Reprints and permission information is available at http://www.nature.com/reprints

Publisher's note Springer Nature remains neutral with regard to jurisdictional claims in published maps and institutional affiliations. 
(c) (i) Open Access This article is licensed under a Creative Commons Attribution 4.0 International License, which permits use, sharing, adaptation, distribution and reproduction in any medium or format, as long as you give appropriate credit to the original author(s) and the source, provide a link to the Creative Commons license, and indicate if changes were made. The images or other third party material in this article are included in the article's Creative Commons license, unless indicated otherwise in a credit line to the material. If material is not included in the article's Creative Commons license and your intended use is not permitted by statutory regulation or exceeds the permitted use, you will need to obtain permission directly from the copyright holder. To view a copy of this license, visit http://creativecommons.org/ licenses/by/4.0/.

(C) The Author(s) 2020 